\title{
32
}

\section{Two Improved Algorithms for Reliability Analysis}

\author{
Y. Zhang ${ }^{1}$ and A. Der Kiureghian ${ }^{2}$
}

${ }^{1}$ Applied Research Associates, Inc., Southeast Division, Raleigh, NC 27615

${ }^{2}$ Department of Civil Engineering, University of California, Berkeley, CA 94720

Improved versions of two optimization algorithms commmonly used in first-order reliability analysis are developed. One is for determining the first-order reliability index $\beta$. The other is for inverse reliability analysis, i.e., determining the value of a deterministic parameter such that the reliability index equals a target value $\beta_{t}$. Besides being mathematically more rigorous, these new versions are much simpler than earlier versions of these algorithms.

\section{Introduction}

Let $G(\boldsymbol{u})$ denote the limit-state function for a reliability problem defined in terms of a vector of standard normal variates, $u$, obtained by a suitable transformation of the basic random variables of the problem. An important quantity of interest is the reliability index defined by

$$
\beta=\min \{\|u\| \mid G(u)=0\}
$$

The above is a constrained optimization problem that can be solved by any of a large number of general-purpose algorithms (Liu and Der Kiureghian 1991). However, there is merit in developing a customized algorithm that takes advantage of the special form of the objective function of this problem. One such algorithm is 'he so called HL-RF algorithm (Hasofer and Lind 1974, Rackwitz and Fiessler 1978, Liu and Der Kiureghian 1991). This algorithm has found wide popularity owing to its simplicity and efficiency. However, there is no proof that for a given problem the algorithm will actually converge. In this paper, an improved version of this algorithm is developed that, under the assumption of differentiability of $G(\boldsymbol{u})$, is globally convergent.

Now suppose the limit-state function includes a deterministic parameter $\theta$, i.e., $G(u, \theta)$. This could be a parameter of the limit-state function in the original space, or it could be a probability distribution parameter that appears in $G(\boldsymbol{u}, \theta)$ due to the transformation of random variables to the standard normal space. The inverse reliability problem is defined as follows: Find $\theta$ such that the reliability index associated with $G(u, \theta)$ equals a target value $\beta_{t}$, i.e.,

$$
\theta: \quad \min \{\|u\| \mid G(u, \theta)=0\}=\beta_{t}
$$

An algorithm for solution of this problem was recently developed by Der Kiureghian et al. (1993). Here, we develop a simpler version of this algorithm that has superior convergence properties. 
The two algorithms developed in this paper have the same format. One generates a sequence of points according to the rule

$$
\boldsymbol{x}_{i+1}=\boldsymbol{x}_{i}+\lambda_{i} \boldsymbol{d}_{i}
$$

where $\boldsymbol{x}_{i}=\boldsymbol{u}_{i}$ for the ordinary reliability problem, $\boldsymbol{x}_{i}=\left(\boldsymbol{u}_{i}, \theta_{i}\right)$ for the inverse reliability problem, $\boldsymbol{d}_{i}$ denotes a search direction vector, and $\lambda_{i}$ is the step size. $\lambda_{i}$ is determined by assuring a reduction in a merit function $m(\boldsymbol{x})$ at each step. Most commonly, the Armijo rule (Luenberger 1986) is used. For the present application, this rule can be written as

$$
\lambda_{i}=\max _{k}\left\{b^{k} \mid m\left(\boldsymbol{x}_{i}+b^{k} \boldsymbol{d}_{i}\right)-m\left(\boldsymbol{x}_{i}\right) \leq-a b^{k}\left\langle\boldsymbol{\nabla} m\left(\boldsymbol{x}_{i}\right), \boldsymbol{d}_{i}\right\rangle\right\},
$$

where $a, b \in(0,1)$ are pre-selected parameters and $k$ is an integer. Global convergence of the sequence in (3) is assured if the merit function attains its minimum at the solution point of the problem and if $\boldsymbol{d}_{i}$ is a descent direction of $m\left(\boldsymbol{x}_{i}\right)$ at every point of the sequence (Luenberger 1986). The main issue is the selection of $\boldsymbol{d}_{i}$ and $m\left(\boldsymbol{x}_{\boldsymbol{i}}\right)$ that satisfy these conditions for each problem.

\section{An Improved HL-RF Algorithm}

The solution of the optimization problem in (1) is characterized by the following optimality conditions:

$$
\begin{gathered}
u+\frac{\|u\|}{\left\|\nabla_{u} G(u)\right\|} \nabla_{u} G(u)=0, \\
G(u)=0,
\end{gathered}
$$

where $\boldsymbol{\nabla}_{\boldsymbol{u}}$ denotes the gradient operator with respect to $\boldsymbol{u}$. In the following, the subscript on the gradient operator is dropped unless it is necessary to avoid ambiguity. The HL-RF search direction is obtained by solving the above equations for the linearized constraint

$$
L_{\boldsymbol{u}_{i}}(\boldsymbol{u})=G\left(\boldsymbol{u}_{i}\right)+\left\langle\nabla G\left(\boldsymbol{u}_{i}\right), \boldsymbol{u}-\boldsymbol{u}_{i}\right\rangle
$$

at each step, where $\langle.,$.$\rangle denotes the inner product of two vectors. The result, dropping$ the subscript $i$ for simplicity, is

$$
\boldsymbol{d}=\frac{\langle\boldsymbol{u}, \boldsymbol{\nabla} G(\boldsymbol{u})\rangle-G(\boldsymbol{u})}{\|\boldsymbol{\nabla} G(\boldsymbol{u})\|^{2}} \nabla G(\boldsymbol{u})-\boldsymbol{u} .
$$

The original HL-RF algorithm (Hasofer and Lind 1974, Rackwitz and Fiessler 1978) used a unit step size, i.e., $\lambda_{i}=1$. Liu and Der Kiureghian (1991) introduced a modified version that has step size control by using the merit function

$$
m(\boldsymbol{u})=\frac{1}{2}\left\|\boldsymbol{u}-\frac{\langle\boldsymbol{u}, \boldsymbol{\nabla} G(\boldsymbol{u})\rangle}{\|\boldsymbol{\nabla} G(\boldsymbol{u})\|^{2}} \boldsymbol{\nabla} G(\boldsymbol{u})\right\|^{2}+\frac{1}{2} c G(\boldsymbol{u})^{2},
$$


where $c>0$ is a constant. This merit function has its minimum at the solution of (1). However, there is no proof that $\boldsymbol{d}$ in (7) is a descent direction of this merit function.

Here, we introduce the simpler merit function

$$
m(\boldsymbol{u})=\frac{1}{2}\|u\|+c|G(\boldsymbol{u})|
$$

where $c>0$ is a penalty parameter. This merit function also attains its minimum at the solution of (1). Furthermore, as shown by the following theorem, the merit function in (9) is compatible with the search direction in (7).

Theorem: The HL-RF search direction $\boldsymbol{d}$ in (7) at any point $\boldsymbol{u}$ is a descent direction of the merit function $m(\boldsymbol{u})$ in (9), provided

$$
c>\frac{\|\boldsymbol{u}\|}{\|\boldsymbol{\nabla} G(\boldsymbol{u})\|} .
$$

Proof: To prove that $\boldsymbol{d}$ is a descent direction of $m(\boldsymbol{u})$, we need to show that $\langle\boldsymbol{\nabla} m(\boldsymbol{u}), \boldsymbol{d}\rangle \leq 0$ and that the equality holds only if $\boldsymbol{u}$ is a solution to (1).

Using (7) together with $\boldsymbol{\nabla} m(\boldsymbol{u})=\boldsymbol{u}+c \operatorname{sgn}(G(\boldsymbol{u})) \boldsymbol{\nabla} G(\boldsymbol{u})$, where $\operatorname{sgn}(G(\boldsymbol{u}))$ is the sign of $G(\boldsymbol{u})$, we have

$$
\begin{aligned}
\langle\boldsymbol{\nabla} m(\boldsymbol{u}), \boldsymbol{d}\rangle= & -\left(\|\boldsymbol{u}\|^{2}-\frac{\langle\boldsymbol{u}, \boldsymbol{\nabla} G(\boldsymbol{u})\rangle^{2}}{\|\boldsymbol{\nabla} G(\boldsymbol{u})\|^{2}}\right) \\
& -|G(\boldsymbol{u})|\left(c+\operatorname{sgn}(G(\boldsymbol{u})) \frac{\langle\boldsymbol{u}, \boldsymbol{\nabla} G(\boldsymbol{u})\rangle}{\|\boldsymbol{\nabla} G(\boldsymbol{u})\|^{2}}\right)
\end{aligned}
$$

Using the Schwartz inequality $|\langle\boldsymbol{u}, \boldsymbol{\nabla} G(\boldsymbol{u})\rangle| \leq\|\boldsymbol{u}\|\|\nabla G(\boldsymbol{u})\|$,

$$
\|u\|^{2}-\frac{\langle u, \nabla G(u)\rangle^{2}}{\|\nabla G(u)\|^{2}} \geq 0 .
$$

Using Schwartz inequality again and (10),

$$
\begin{aligned}
c+\operatorname{sgn}(G(u)) \frac{\langle\boldsymbol{u}, \boldsymbol{\nabla} G(\boldsymbol{u})\rangle}{\|\boldsymbol{\nabla} G(\boldsymbol{u})\|^{2}} & \geq c-\frac{|\langle\boldsymbol{u}, \boldsymbol{\nabla} G(\boldsymbol{u})\rangle|}{\|\boldsymbol{\nabla} G(\boldsymbol{u})\|^{2}} \\
& \geq c-\frac{\|\boldsymbol{u}\|}{\|\boldsymbol{\nabla} G(\boldsymbol{u})\|} \\
& >0 .
\end{aligned}
$$

Using (12) and (13) in (11), it follows that $\langle\nabla m(\boldsymbol{u}), \boldsymbol{d}\rangle \leq 0$. If $\langle\boldsymbol{\nabla} m(\boldsymbol{u}), \boldsymbol{d}\rangle=0$, we have from (11)-(13) that $G(\boldsymbol{u})=0$ and $\|\boldsymbol{u}\|^{2}-\frac{\langle\boldsymbol{u} \nabla G(\boldsymbol{u})\rangle^{2}}{\|\nabla G(\boldsymbol{u})\|^{2}}=0$, which are equivalent to the 
optimality conditions (5). Hence, in this case $u$ is a solution to (1), which completes the proof.

The above theorem is an essential element for establishing the global convergence theorem. The mathematical analysis and poof of the global convergence of the sequence along with a proper line search scheme, can be found in standard texts on optimization theory (Luenberger 1986). In implementing this algorithm, it is suggested to gradually increase the value of the penalty parameter $c$ while satisfying (10).

The proposed algorithm is superior to the modified HL-RF algorithm of Liu and Der Kiureghian (1991) in at least two ways: (a) there is a mathematical proof that the merit function is compatible with the search direction, and (b) the algorithm usually requires less computation since the merit function in (9) does not involve the gradient of the constraint function as does the merit function in (8).

Below, we present two examples comparing the performance of the modified HL-RF algorithm of Liu and Der Kiureghian (1991) with the proposed algorithm.

Example 1 Consider the original-space, limit-state function

$$
\begin{aligned}
g(\boldsymbol{z}) & =1.1-0.00115 z_{1} z_{2}+0.00157 z_{2}^{2}+0.00117 z_{1}^{2}+0.0135 z_{2} z_{3}-0.0705 z_{2} \\
& -0.00534 z_{1}-0.0149 z_{1} z_{3}-0.0611 z_{2} z_{4}+0.0717 z_{1} z_{4}-0.226 z_{3} \\
& +0.0333 z_{3}^{2}-0.558 z_{3} z_{4}+0.998 z_{4}-1.339 z_{4}^{2},
\end{aligned}
$$

where $z_{1}, z_{2}, z_{3}$ and $z_{4}$ are statistically independent random variables: $z_{1}$ has a type-II largest value distribution with mean 10 and standard deviation $5 ; z_{2}$ and $z_{3}$ are both normal with means 25 and 0.8 and standard deviations 5 and 0.2 , respectively, and $z_{4}$ has the lognormal distribution with mean 0.0625 and standard deviation 0.0625 . The original HL-RF algorithm with $\lambda_{i}=1$ fails to converge for this problem.

Example 2 This example concerns the deformation of an elastic plate subjected to a uniformly distributed edge load of intensity $p=1.0 G P a$, as shown in Fig. 1. The plate is in a state of plane stress condition. The Poisson's ratio is $\nu=0.3$. However, the Young's modulus, $E$, is a Gaussian random field with mean $200 G P a$, standard deviation $50 G P a$, and auto-correlation coefficient function $\rho_{E E}=\exp \left(-\left(\frac{\Delta}{b}\right)^{2}\right)$, where $\Delta$ is the distance between any two points on the plate and $b=2 \mathrm{~cm}$ is the correlation length. The random field is discretized by using the finite element mesh shown in Fig. 1 and is represented by 20 random variables. Further details can be found in Zhang (1994).

The original-space limit-state function for this example is defined by

$$
g(\boldsymbol{z})=\theta-U_{A}(\boldsymbol{z}),
$$

where $z$ denotes the vector of random variables representing the random field of $E$, $\theta=0.19 \mathrm{~cm}$ is the displacement threshold, and $U_{A}(\boldsymbol{z})$ denotes the displacement at 


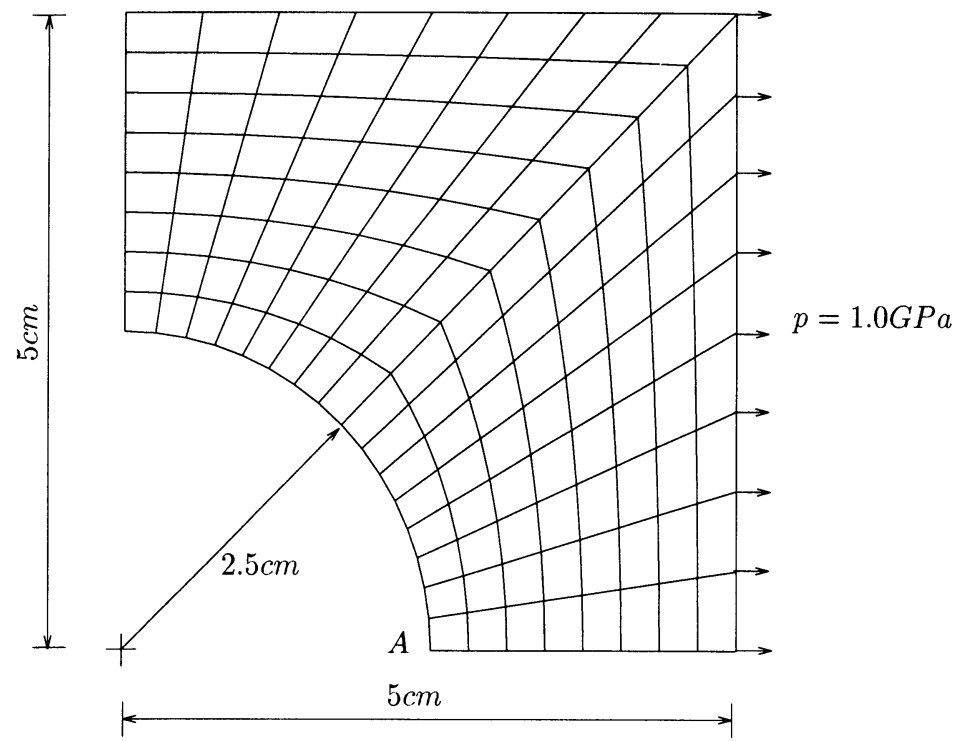

Figure 3.1 Finite element mesh for elastic plate

point $A$ in the horizontal direction. The finite element method is used to compute the limit-state function and its gradient.

Table 1 compares the performance of the two algorithms for the above two examples. The algorithms are initiated at the mean point and tolerances are set at 0.001 . Specification of other parameters is described in Zhang (1994). The two algorithms converge to identical solutions with reliability indices $\beta=1.35$ and $\beta=3.27$ for the two problems, respectively. The required number of steps as well as the number of times each algorithm computed the limit-state function and its gradient are listed in Table 1. For both examples, the proposed algorithm performs significantly better than the modified HL-RF algorithm of Liu and Der Kiureghian (1991).

\section{Inverse Reliability Algorithm}

The inverse reliability problem in (2) is defined by the set of equations

$$
\begin{gathered}
\|u\|-\beta_{t}=0, \\
u+\frac{\|u\|}{\left\|\nabla_{u} G(u, \theta)\right\|} \nabla_{u} G(u, \theta)=0,
\end{gathered}
$$


Table 1. Comparison between two algorithms

\begin{tabular}{|c|c|c|c|c|}
\hline Example & Algorithm & No. steps & No. $g(\boldsymbol{z})$ & No. $\boldsymbol{\nabla} g(\boldsymbol{y})$ \\
\hline \multirow{2}{*}{1} & proposed & 6 & 17 & 7 \\
\cline { 2 - 5 } & modified HL-RF & 8 & 15 & 15 \\
\hline \multirow{2}{*}{2} & proposed & 3 & 8 & 4 \\
\cline { 2 - 5 } & modified HL-RF & 16 & 21 & 21 \\
\hline
\end{tabular}

$$
G(u, \theta)=0,
$$

The last two equations are the optimality conditions of (1), whereas the first equation describes the constraint on the reliability index. A search direction $\boldsymbol{d}_{i}$ for this problem can be derived from the solution of the linearized equations

$$
\begin{gathered}
\|u\|-\beta_{t}=0, \\
u+\frac{\|u\|}{\left\|\nabla_{u} L_{\boldsymbol{u}_{i}, \theta_{i}}(\boldsymbol{u}, \theta)\right\|} \nabla_{u} L_{\boldsymbol{u}_{i}, \theta_{i}}(u, \theta)=0 \\
L_{\boldsymbol{u}_{i}, \theta_{i}}(\boldsymbol{u}, \theta)=0
\end{gathered}
$$

where

$$
L_{\boldsymbol{u}_{i}, \theta_{i}}(\boldsymbol{u}, \theta)=G\left(\boldsymbol{u}_{i}, \theta_{i}\right)+\left\langle\nabla_{\boldsymbol{u}} G\left(\boldsymbol{u}_{i}, \theta_{i}\right), \boldsymbol{u}-\boldsymbol{u}_{i}\right\rangle+\frac{\partial G\left(\boldsymbol{u}_{i}, \theta_{i}\right)}{\partial \theta}\left(\theta-\theta_{i}\right)
$$

The solution of (17) is

$$
\begin{gathered}
u=-\beta_{t} \frac{\nabla_{\boldsymbol{u}} G\left(\boldsymbol{u}_{i}, \theta_{i}\right)}{\left\|\nabla_{\boldsymbol{u}} G\left(\boldsymbol{u}_{i}, \theta_{i}\right)\right\|}, \\
\theta=\theta_{i}+\frac{\left\langle\boldsymbol{u}_{i}, \boldsymbol{\nabla}_{\boldsymbol{u}} G\left(\boldsymbol{u}_{i}, \theta_{i}\right)\right\rangle-G\left(\boldsymbol{u}_{i}, \theta_{i}\right)+\beta_{t}\left\|\boldsymbol{\nabla}_{\boldsymbol{u}} G\left(\boldsymbol{u}_{i}, \theta_{i}\right)\right\|}{\partial G\left(\boldsymbol{u}_{i}, \theta_{i}\right) / \partial \theta} .
\end{gathered}
$$

Using this result, the search direction is obtained as

$$
\boldsymbol{d}_{i}=\left(\begin{array}{c}
-\beta_{t} \frac{\nabla_{u} G\left(\boldsymbol{u}_{i}, \theta_{i}\right)}{\left\|\nabla_{\boldsymbol{u}} G\left(\boldsymbol{u}_{i}, \theta_{i}\right)\right\|}-\boldsymbol{u}_{i} \\
\frac{\left\langle\boldsymbol{\nabla}_{\boldsymbol{u}} G\left(\boldsymbol{u}_{i}, \theta_{i}\right), \boldsymbol{u}_{i}\right\rangle-G\left(\boldsymbol{u}_{i}, \theta_{i}\right)+\beta_{t}\left\|\boldsymbol{\nabla}_{\boldsymbol{u}} G\left(\boldsymbol{u}_{i}, \theta_{i}\right)\right\|}{\partial G\left(\boldsymbol{u}_{i}, \theta_{i}\right) / \partial \theta}
\end{array}\right)
$$

With the above direction vector, a full step size (i.e., with $\lambda_{i}=1$ ) entirely satisfies (16a). Hence, any step size $0<\lambda_{i} \leq 1$ along $\boldsymbol{d}_{i}$ would be favorable to (16a). We need to select a step size such that the move is also favorable to (16b) and (16c). Since for 
a fixed $\theta$ these equations are identical to (5a) and (5b), we consider a merit function similar to that used for the previous algorithm, i.e.,

$$
m(\boldsymbol{u}, \theta)=\frac{1}{2}\|\boldsymbol{u}\|^{2}+c|G(\boldsymbol{u}, \theta)|
$$

This merit function is compatible with the search direction in $(20)$ for $|G(u, \theta)|>0$ (points not on the limit-state surface) and $c>\beta_{t}\|u\| /|G(u, \theta)|$. To see this, we examine $\langle\boldsymbol{\nabla} m(\boldsymbol{u}, \theta), \boldsymbol{d}\rangle<0$ by using $(20)$ and

$$
\boldsymbol{\nabla} m(\boldsymbol{u}, \theta)=\left(\begin{array}{l}
\boldsymbol{u} \\
0
\end{array}\right)+c \operatorname{sgn}(G(\boldsymbol{u}, \theta))\left(\begin{array}{c}
\boldsymbol{\nabla}_{\boldsymbol{u}} G(\boldsymbol{u}, \theta) \\
\frac{\partial G(\boldsymbol{u}, \theta)}{\partial \theta}
\end{array}\right) .
$$

We have

$$
\begin{aligned}
\langle\boldsymbol{\nabla} m(\boldsymbol{u}, \theta), \boldsymbol{d}\rangle & =-\|\boldsymbol{u}\|^{2}-\frac{\beta_{t}}{\left\|\boldsymbol{\nabla}_{\boldsymbol{u}} G(\boldsymbol{u}, \theta)\right\|}\left\langle\boldsymbol{u}, \boldsymbol{\nabla}_{\boldsymbol{u}} G(\boldsymbol{u}, \theta)\right\rangle-c|G(\boldsymbol{u}, \theta)| \\
& <-\|\boldsymbol{u}\|^{2}-\beta_{t}\|\boldsymbol{u}\|\left(1+\left\langle\frac{\boldsymbol{u}}{\|\boldsymbol{u}\|}, \frac{\boldsymbol{\nabla}_{\boldsymbol{u}} G(\boldsymbol{u}, \theta)}{\left\|\boldsymbol{\nabla}_{\boldsymbol{u}} G(\boldsymbol{u}, \theta)\right\|}\right\rangle\right) \\
& <-\|\boldsymbol{u}\|^{2} .
\end{aligned}
$$

Unfortunately, we cannot ascertain that the minimum of $m(\boldsymbol{u}, \theta)$ in $(21)$ coincides with the solution of (16). Hence, global convergence of the algorithm cannot be proven. Nevertheless, based on the above analysis and extensive numerical testing, we find that the merit function in (21) is effective in stabilizing the algorithm. In actual implementation, it is necessary to set $c>\beta_{t}\|u\| / \delta$, where $\delta$ denotes the tolerance in satisfying (16c).

The above algorithm for inverse reliability analysis is superior to the earlier algorithm by Der Kiureghian et al. (1993) for two reasons: (a) it is computationally simpler as the merit function does not involve the gradient, (b) the search direction is a descent direction of the merit function under the conditions specified earlier. Below, we present two examples to demonstrate the proposed inverse reliability algorithm.

Example 3 - The example is defined by the limit-state function in the standard normal space

$$
G(\boldsymbol{u}, \theta)=\exp \left(-\theta\left(u_{1}+2 u_{2}+3 u_{3}\right)\right)-u_{4}+1.5 .
$$

For $\beta_{t}=2$, and starting from the initial point $\left(u_{0}, \theta_{0}\right)=(0.2,0.2,0.2,0.2,0.1)$, the proposed algorithm converges in 4 steps as shown in Table 2.

Example 4 - Reconsider Example 2 defined by the limit-state function (15). Assuming a target reliability index $\beta_{t}=3.0$, we wish to determine the corresponding threshold for the displacement at point $A$. Starting from $\theta_{0}=0.1 \mathrm{~cm}$ and $z_{0}$ at the mean point, the algorithm converges in 5 steps yielding the solution $\theta=0.172 \mathrm{~cm}$. 
Table 2. Convergence of the inverse reliability algorithm

\begin{tabular}{|c|c|c|c|}
\hline Step & $\boldsymbol{u}$ & $\theta$ & $\|\boldsymbol{u}\|$ \\
\hline 0 & $(0.200,0.200,0.200,0.200)$ & 0.100 & 0.400 \\
1 & $(0.168,0.337,0.505,1.898)$ & 0.463 & 2.000 \\
2 & $(0.219,0.437,0.656,1.813)$ & 0.392 & 1.989 \\
3 & $(0.216,0.432,0.648,1.829)$ & 0.366 & 2.000 \\
4 & $(0.220,0.441,0.661,1.822)$ & 0.367 & 2.000 \\
\hline
\end{tabular}

\section{Conclusion}

Improved versions of two popular algorithms for first-order reliability analysis are presented. One is an improved version of the HL-RF algorithm for determining the reliability index defined as the nearest point from the origin to the limit-state surface in the standard normal space. A proof of global convergence for this algorithm is provided. The other is an inverse reliability algorithm used for determining a deterministic parameter in the limit-state function such that the reliability index equals a target value. Although proof of global convergence for this algorithm is not presented, the algorithm possesses superior convergence properties than previously available. Both proposed algorithms are mathematically more rigorous, yet simpler and more efficient than existing similar algorithms.

\section{References}

[1] Der Kiureghian, A., Zhang, Y. and Li, C.-C., "Inverse reliability problem," J. Eng. Mech., ASCE, 120(5), 1154-1159, 1994.

[2] Hasofer, A. M. and Lind, N. C., "Exact and invariant second-moment code format," J. Eng. Mech. Div., 100(EM1),111 121, 1974.

[3] Liu, P.-L. and Der Kiureghian, A., "Optimization algorithms for structural reliability," Structural Safety, 9(3), 161-177, 1991.

[4] Luenberger, D. G., Introduction to Linear and Nonlinear Programming, Addison Wesley, Reading, Massachusetts, 1986.

[5] Rackwitz, R. and Fiessler, B., "Structural reliability under combined random load sequences," Computers \& Structures, 9, 489-494, 1978.

[6] Zhang,Y., "Finite element reliability methods for inelastic structures." Thesis submitted in partial satisfaction of the requirements for the Ph.D. degree, Department of Civil Engineering, University of California, Berkeley, CA, 1994. 